\title{
Update zum Zulassungsstopp - Inkraftsetzung per 1. Juli 2013
}

Das Parlament hat in der Sommersession 2013 einen erneuten Zulassungsstopp beschlossen. Er betrifft Ärztinnen und Ärzte, die ihre Tätigkeit selbständig oder unselbständig in der freien Praxis ausüben wollen oder in einer Einrichtung bzw. im ambulanten Bereich von Spitälern tätig sein möchten. Der Zulassungsstopp gilt neu sowohl für Grundversorger als auch für Spezialistinnen. Gemäss dringlichem Bundesrecht tritt er per 1. Juli 2013 in Kraft und dauert bis zum 30. Juni 2016. Es liegt jedoch in den Händen der Kantone, ob sie die Zulassung zur Obligatorischen Krankenpflegeversicherung auch tatsächlich von einem Bedürfnis abhängig machen wollen.

\author{
Hanspeter Kuhn ${ }^{a}$, \\ Gabriela Lang ${ }^{b}$ \\ a Fürsprecher, \\ Leiter Rechtsdienst, \\ stv. Generalsekretär der FMH \\ b Rechtsanwältin, \\ Rechtsdienst FMH
}

\section{Nicht betroffen vom Zulassungsstopp}

- sind Ärztinnen und Ärzte aller Fachrichtungen, die schon vor dem 1. Juli 2013 in eigener Praxis zulasten der Obligatorischen Krankenpflegeversicherung tätig waren (d.h. in eigenem Namen und auf eigene Verantwortung Patienten behandelt haben).

- sind Ärztinnen und Ärzte, die alle nötigen Schritte für die Praxiseröffnung bis und mit vollständigem Antrag zur Erteilung der ZSRNummer unternommen haben und infolge Verspätung der ZSR-Nummern-Erteilung am 1. Juli 2013 die Tätigkeit noch nicht aufgenommen haben.

- sind Ärztinnen und Ärzte aller Fachrichtungen, die mindestens drei Jahre an einer anerkannten schweizerischen Weiterbildungsstätte gearbeitet haben.

\section{«Zulasten der obligatorischen Krankenpflegeversicherung tätig waren"} Der Gesetzestext hält fest: Nicht betroffen vom Zulassungsstopp sind Ärztinnen und Ärzte, die vor dem 1. Juli 2013 «in eigener Praxis zulasten der obligatorischen Krankenpflegeversicherung tätig waren». Zulasten der Sozialversicherung tätig ist, wer einen eidgenössischen oder einen anerkannten Facharzttitel (oder den Weiterbildungstitel praktischer Arzt) hat, über eine kantonale Berufsausübungsbewilligung und eine Haftpflichtversicherung verfügt, den Beitritt zum Rahmenvertrag TARMED und zu den kantonalen Anschlussverträgen erklärt hat und in eigenem Namen und eigener Verantwortung KVGPatienten behandelt hat.

\section{Betroffen vom Zulassungsstopp}

- sind Ärztinnen und Ärzte aller Fachrichtungen, die vor dem 1. Juli 2013 weder selbständig zulasten der Obligatorischen Krankenpflegeversicherung tätig waren, noch drei Jahre an einer anerkannten schweizerischen Weiterbildungsstätte gearbeitet haben.

\section{Ausnahmen:}

Vom Zulassungsstopp gemäss KVG betroffene Ärztinnen und Ärzte können in die selbständige Praxis gehen, wenn

- der Kanton den Zulassungsstopp nicht anwendet

- sie vom Kanton eine eigene KVG-Zulassung erteilt bekommen;

- sie eine bestehende Arztpraxis und damit die KVG-Zulassung des Praxisübergebers übernehmen können [1].

\section{Alle nötigen Schritte unternommen, aber wegen ZSR-Verzögerung noch nicht tätig} Um abrechnen zu können, ist gemäss Rahmenvertrag TARMED eine ZSR-Nummer notwendig. Im Hinblick auf einen möglichen erneuten Zulassungsstopp wurde deshalb in den vergangenen Monaten bei der SASIS AG vermehrt eine ZSR-Nummer beantragt. Dies führte zu grossen Verzögerungen bei der Erteilung der ZSR-Nummer. Dieser Umstand war auch im Parlament bekannt und führte dazu, dass Bundesrat Alain Berset an der Sitzung des Ständerates zuhanden des Protokolls festhielt: «(...) Mais je souhaitais dire clairement ici qu'il ne saurait être envisageable qu'un médecin, qui a tout fait dans le temps pour obtenir un numéro, parce qu'il y a un 


\section{Interaktiver \\ Artikel \\ 3-jährige Tätigkeit}

Wollen Sie diesen Artikel kommentieren? Nutzen Sie dafür die Kommentarfunktion in der OnlineVersion oder sehen Sie nach, was Ihre Kolleginnen und Kollegen bereits geschrieben haben: www.saez.ch/ aktuelle-ausgabe/ interaktive-beitraege/ retard dû à l'institution qui les distribue, ne puisse pas s'installer; nous souhaitons que ce soit possible. C'est aussi une réponse à une proposition qui avait été déposée en commission par Monsieur Graber; je souhaitais préciser cela au conseil (...)» [2].

Aufgrund des Votums von Bundesrat Berset muss es genügen, wenn die Ärztin oder der Arzt vor dem 1. Juli 2013 die nötigen Schritte unternommen hat für die Praxiseröffnung bis und mit Antrag für die ZSR-Nummer. Trifft die ZSR-Nummer verspätet ein, ist die Praxiseröffnung dennoch möglich. $\mathrm{Zu}$ den nötigen Schritten im Sinn der Protokollbemerkung von Bundesrat Berset gehört, vor dem 1. Juli 2013

- im Besitz einer kantonalen Berufsausübungsbewilligung zu sein,

- eine Berufshaftpflichtversicherung zu haben, dem Rahmenvertrag TARMED und dem kantonalen Anschlussvertrag beigetreten zu sein und

- den Antrag auf eine ZSR-Nummer gestellt zu haben.

Nicht betroffen vom Zulassungsstopp sind Ärztinnen und Ärzte aller Fachrichtungen, die mindestens drei Jahre an einer anerkannten schweizerischen Weiterbildungsstätte gearbeitet haben, und zwar unabhängig davon, ob sich jemand während dieser drei Jahre in Weiterbildung befand oder als Facharzt tätig war. Die 3-jährige Tätigkeit braucht auch nicht an einem Stück zu erfolgen und sie muss nicht bereits vor Einführung des Zulassungsstopps vollständig absolviert worden sein. Sie muss auf den Zeitpunkt nachgewiesen werden, in welchem Ärztinnen und Ärzte in die selbständige Praxis wollen, sich in einer Praxis anstellen lassen möchten oder eine Tätigkeit im ambulanten Bereich eines Spitals anstreben.

\section{Anerkannte Weiterbildungsstätten}

Die anerkannten Weiterbildungsstätten in der Schweiz findet man über das entsprechende Register des Schweizerischen Instituts für ärztliche Weiterund Fortbildung SIWF: www.siwf-register.ch

\section{Nachweis der 3-jährigen Tätigkeit}

Die erforderliche 3-jährige Tätigkeit in der Schweiz kann z.B. nachgewiesen werden

- durch SIWF/FMH-Zeugnisse, welche während der Weiterbildung zum Facharzt von der anerkannten schweizerischen Weiterbildungsstätte ausgestellt wurden oder

- durch Arbeitsverträge mit anerkannten schweizerischen Weiterbildungsstätten.

Unzureichend ist, nur den schweizerischen oder den anerkannten ausländischen Facharzttitel vorzulegen.

\section{Referenzen}

1 Praxisübernahmen sollten wie unter dem alten Zulassungsstopp seitens der Kantone ohne Schwierigkeiten möglich sein, sofern es sich um die gleiche Fachrichtung handelt. Wenn eine Praxisübernahme geplant ist, sollte sich der Übernehmer vor Unterzeichnung des Praxisübernahmevertrages versichern, dass der Übergeber beim Kanton abgeklärt hat, dass er seine Zulassung auch wirklich auf einen Nachfolger übertragen lassen kann.

2 Amtliches Bulletin, Sitzung des Ständerates vom 5.6.2013. www.parlament.ch/ab/

frameset/d/s/4909/406719/d_s_4909_406719_406737. htm

\section{Bringt Vorteile. Die Mitgliedschaft in der FMH.}

Wir machen uns stark für Sie. Mit engagierter Standespolitik und attraktiven Services: TarifInfoline, Rechtsauskünfte, Musterverträge.

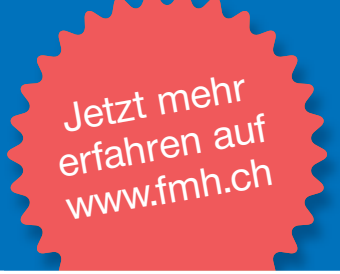

